\title{
Outer Synchronization of Complex-Variable Networks with Complex Coupling via Impulsive Pinning Control
}

\author{
Yanjie Ji and Zhaoyan $\mathrm{Wu} *$ (D) \\ School of Mathematics and Statistic, Jiangxi Normal University, Nanchang 330022, China; \\ 20194010047@jxnu.edu.cn \\ * Correspondence: zhywu@jxnu.edu.cn; Tel.: +86-791-8812-0367
}

check for updates

Citation: Ji, Y.; Wu, Z. Outer Synchronization of Complex-Variable Networks with Complex Coupling via Impulsive Pinning Control. Mathematics 2021, 9, 2110. https:// doi.org/10.3390/math9172110

Received: 22 June 2021

Accepted: 25 August 2021

Published: 1 September 2021

Publisher's Note: MDPI stays neutral with regard to jurisdictional claims in published maps and institutional affiliations.

Copyright: (c) 2021 by the authors. Licensee MDPI, Basel, Switzerland. This article is an open access article distributed under the terms and conditions of the Creative Commons Attribution (CC BY) license (https:// creativecommons.org/licenses/by/ $4.0 /)$.

\begin{abstract}
In this paper, outer synchronization of complex-variable networks with complex coupling is considered. Sufficient conditions for achieving outer synchronization using static impulsive pinning controllers are first derived according to the Lyapunov function method and stability theory of impulsive differential equations. From these conditions, the necessary impulsive gains and intervals for given networks can be easily calculated. Further, an adaptive strategy is introduced to design universal controllers and avoid repeated calculations for different networks. Notably, the estimation algorithms of the impulsive gains and intervals are provided. Finally, three numerical examples are performed to verify the effectiveness of the main results.
\end{abstract}

Keywords: outer synchronization; dynamical network; complex variable; impulsive pinning control

\section{Introduction}

Outer synchronization between two networks is an important phenomenon and exists widely in real-world situations [1-12]. In [1], authors established a public traffic supernetwork model, which consists in the conventional bus traffic network and the urban rail traffic network. They then studied the impact of the departing frequencies of both types of public traffic vehicles, and the coordinated scheduling in the process of transfer to the supernetwork model's synchronous ability by using outer synchronization. In [2], authors discussed the applications of outer synchronization of anti-star networks in secure communication. In [3], authors studied the outer synchronization and parameter identification of supply networks with uncertainty. They also used the obtained results for resilient recovery. In [4], authors studied the generalized outer synchronization non-dissipatively with different-dimensional nodes. In [5-8], authors studied the outer synchronization of fractional-order dynamical networks. In [9], authors studied the outer synchronization of time-varying networks with different node numbers.

Due to the complexities of dynamical networks, outer synchronization between them is difficult and even impossible to achieve without external control. Therefore, many control schemes are adopted to design effective controllers, such as impulsive control [13,14], intermittent control [15,16], pinning control [14-17], finite-time control [18], and so on. In [14], authors considered the outer synchronization of drive-response networks by combining impulsive and pinning control. It is noted that adaptive strategies are introduced to design universal controllers as well. In $[15,16]$, authors investigated the outer synchronization via intermittent pinning control. In [17], authors considered pinning outer synchronization. In [18], authors considered finite-time outer synchronization and discussed its application in image encryption.

Nevertheless, the above-mentioned results mainly concentrate on dynamical networks coupled with real-variable systems. Alternatively, complex-variable systems are employed to describe physical systems [19-24]. In [22], authors used a complex-variable Lorenz system to describe rotating fluid. In [23], authors simulated detuned lasers using a complexvariable Lorenz system. In [19], authors applied complex-valued convolutional neural 
network in polarimetric SAR image classification. Naturally, dynamical networks coupled with complex-variable systems have drawn increasing attention from researchers and many valuable results have been obtained [25-36]. In [35], authors studied the outer synchronization of drive-response networks via intermittent pinning control. In [36], authors considered complex-variable networks with both real and complex coupling, which can better describe the interactions. Until now, there have been few results about outer synchronization of complex-variable networks with complex coupling via impulsive pinning control.

By virtue of the advantages of impulsive and pinning control schemes, in this paper, we investigate the outer synchronization of complex-variable networks with complex coupling through combining impulsive and pinning control. The main contributions are twofold:

(i) We design the static impulsive pinning controllers and provide the method for choosing pinned nodes based on the norm of synchronization errors. According to the Lyapunov function method and stability theory of impulsive differential equations, we derive the synchronization criteria, from which we can calculate the necessary impulsive gains and intervals for any given networks.

(ii) We introduce an adaptive strategy to design universal controllers for different networks. The designed controllers can avoid the repeated calculations of the impulsive gains and intervals. That is, the impulsive instants can be adaptively estimated according to the updating laws (see Remark 2).

The rest of this paper is organized as follows. In Section 2, we introduce the network model and some preliminaries. In Section 3, we derive two main results. We perform three numerical examples to illustrate our main results in Section 4. Finally, we conclude this paper in Section 5.

\section{Model Description and Preliminaries}

Consider a complex-variable dynamical network consisting of $N$ nodes with complex coupling, which is regarded as the drive network and described by

$$
\dot{x}_{k}(t)=f\left(x_{k}(t)\right)+c \sum_{l=1}^{N} a_{k l} H_{1} x_{l}(t)+c \sum_{l=1}^{N} b_{k l} H_{2} x_{l}(t), k=1,2, \cdots, N,
$$

where $x_{k}(t)=\left(x_{k 1}(t), \cdots, x_{k, m+n}(t)\right)^{T}$ is the state variable, $\left(x_{k 1}(t), \cdots, x_{k m}(t)\right)^{T} \in C^{m}$ and $\left(x_{k, m+1}(t), \cdots, x_{k, m+n}(t)\right)^{T} \in R^{n}$ are the complex and real components, respectively, $f$ is a continuous and differentiable vector-valued function, $c>0$ is the coupling strength, $H_{1}=$ $\operatorname{diag}(\underbrace{h_{1}^{1}, \cdots, h_{1}^{m}}_{m}, \underbrace{0, \cdots, 0}_{n})$ and $H_{2}=\operatorname{diag}(\underbrace{0, \cdots, 0}_{m}, \underbrace{h_{2}^{1}, \cdots, h_{2}^{n}}_{n})$ denote the inner coupling matrices. $A=\left(a_{k l}\right) \in C^{N \times N}$ and $B=\left(b_{k l}\right) \in R^{N \times N}$ are the zero-row-sum outer coupling matrices. If there exists a connection from node $l$ to node $k$, then $a_{k l} \neq 0$ and $b_{k l} \neq 0(k \neq l)$, otherwise, $a_{k l}=b_{k l}=0$.

The corresponding response network with impulsive controllers is described by

$$
\begin{aligned}
& \dot{y}_{k}(t)=f\left(y_{k}(t)\right)+c \sum_{l=1}^{N} a_{k l} H_{1} y_{l}(t)+c \sum_{l=1}^{N} b_{k l} H_{2} y_{l}(t), \quad t \neq t_{\sigma}, \\
& y_{k}\left(t_{\sigma}^{+}\right)=y_{k}\left(t_{\sigma}^{-}\right)+b_{k}\left(t_{\sigma}\right)\left(y_{k}\left(t_{\sigma}^{-}\right)-x_{k}\left(t_{\sigma}^{-}\right)\right), \quad t=t_{\sigma},
\end{aligned}
$$

where $k=1,2, \cdots, N, \sigma=1,2, \cdots, y_{k}(t)=\left(y_{k 1}(t), \cdots, y_{k, m+n}(t)\right)^{T}$ is the response state vector of the node $k,\left(y_{k 1}(t), \cdots, y_{k, m}(t)\right)^{T} \in C^{m}$ and $\left(y_{k, m+1}(t), \cdots, y_{k, m+n}(t)\right)^{T} \in R^{n}$ are the complex and real components, the impulsive instants $t_{\sigma}$ satisfies $0=t_{0}<t_{1}<$ $t_{2}<\cdots<t_{\sigma}<\cdots$, and $t_{\sigma} \rightarrow+\infty$ as $\sigma \rightarrow+\infty, y_{k}\left(t_{\sigma}^{+}\right)=\lim _{t \rightarrow t_{\sigma}^{+}} y_{k}(t)$ and $y_{k}\left(t_{\sigma}^{-}\right)=$ $\lim _{t \rightarrow t_{\sigma}^{-}} y_{k}(t), b_{k}\left(t_{\sigma}\right)$ is the impulsive gain at $t=t_{\sigma}$, and $b_{k}(t)=0$ for $t \neq t_{\sigma}$. The solutions of (2) are piecewise left continuous at $t_{\sigma}$, i.e., $y_{k}\left(t_{\sigma}^{-}\right)=y_{k}\left(t_{\sigma}\right)$. 
Definition 1. The drive-response networks (1) and (2) are said to achieve outer synchronization if

$$
\lim _{t \rightarrow+\infty}\left\|y_{k}(t)-x_{k}(t)\right\|=0, k=1,2, \cdots, N
$$

Let $e_{k}(t)=y_{k}(t)-x_{k}(t)$ be the synchronization errors, then we have the following error systems:

$$
\begin{aligned}
& \dot{e}_{k}(t)=f\left(y_{k}(t)\right)-f\left(x_{k}(t)\right)+c \sum_{l=1}^{N} a_{k l} H_{1} e_{l}(t)+c \sum_{l=1}^{N} b_{k l} H_{2} e_{l}(t), t \neq t_{\sigma}, \\
& e_{k}\left(t_{\sigma}^{+}\right)=e_{k}\left(t_{\sigma}^{-}\right)+b_{k}\left(t_{\sigma}\right) e_{k}\left(t_{\sigma}^{-}\right), \quad t=t_{\sigma} .
\end{aligned}
$$

When $t=t_{\sigma}$, arrange $e_{k}(t)$ as

$$
\left\|e_{k_{1}(t)}(t)\right\| \geq\left\|e_{k_{2}(t)}(t)\right\| \geq \cdots \geq\left\|e_{k_{p}(t)}(t)\right\| \geq\left\|e_{k_{p+1}(t)}(t)\right\| \geq \cdots \geq\left\|e_{k_{N}(t)}(t)\right\|,
$$

where $k_{p}(t) \in\{1,2, \cdots, N\}, p=1,2, \cdots, N$, and if $p \neq q$, then $k_{p}(t) \neq k_{q}(t)$. Further, if $\left\|e_{k_{p}(t)}(t)\right\|=\left\|e_{k_{p+1}(t)}(t)\right\|$, then $k_{p}(t)<k_{p+1}(t)$. Let $P\left(t_{\sigma}\right)=\left\{k_{1}\left(t_{\sigma}\right), k_{2}\left(t_{\sigma}\right), \cdots, k_{p}\left(t_{\sigma}\right)\right\}$ be a set of $p$ nodes. If $k \in P\left(t_{\sigma}\right)$, then $b_{k}\left(t_{\sigma}\right)=b \in(-2,-1) \cup(-1,0)$, otherwise, $b_{k}\left(t_{\sigma}\right)=0$, which means that $P\left(t_{\sigma}\right)$ is the index set of pinned nodes at $t=t_{\sigma}$.

Assumption 1. Suppose that there exists a positive constant $M>0$ such that the function $f(x)$ satisfies

$$
\overline{(y-x)^{T}}(f(y)-f(x))+\overline{(f(y)-f(x))^{T}}(y-x) \leq M \overline{(y-x)^{T}}(y-x) .
$$

\section{Main Results}

Let $e(t)=\left(e_{1}^{T}(t), \cdots, e_{N}^{T}(t)\right)^{T}, \tau_{\sigma}=t_{\sigma}-t_{\sigma-1}$ be the impulsive intervals, $\lambda$ be the largest eigenvalue of $c\left(A \otimes H_{1}+\overline{A^{T}} \otimes \overline{H_{1}}\right)+c\left(B+B^{T}\right) \otimes H_{2}, \beta=(1+b)^{2}, \rho\left(t_{\sigma}\right)=$ $1-p(1-\beta) / N$ for $t=t_{\sigma}$ and $\rho(t)=1$ for $t \neq t_{\sigma}$.

Theorem 1. Suppose that Assumption 1 holds. If there exists a positive constant $\zeta$ such that

$$
\ln \rho\left(t_{\sigma}\right)+\zeta+(M+\lambda) \tau_{\sigma}<0, \sigma=1,2, \cdots,
$$

holds, then the drive-response networks (1) and (2) achieve the outer synchronization.

Proof. Consider the following Lyapunov function:

$$
V(t)=\sum_{k=1}^{N} \overline{e_{k}^{T}(t)} e_{k}(t),
$$

for $t \in\left(t_{\sigma-1}, t_{\sigma}\right], \sigma=1,2, \cdots$. 
When $t \in\left(t_{\sigma-1}, t_{\sigma}\right)$,

$$
\begin{aligned}
\dot{V}(t)= & \sum_{k=1}^{N} \overline{e_{k}^{T}(t)} \dot{e}_{k}(t)+\sum_{k=1}^{N} \overline{\dot{e}_{k}^{T}(t)} e_{k}(t) \\
= & \left.\sum_{k=1}^{N} \overline{\left(e_{k}^{T}(t)\right.}\left(f\left(y_{k}(t)\right)-f\left(x_{k}(t)\right)\right)+\overline{\left(f\left(y_{k}(t)\right)-f\left(x_{k}(t)\right)\right)^{T}} e_{k}(t)\right) \\
& +c \sum_{k=1}^{N} \sum_{l=1}^{N}\left(a_{k l} \overline{e_{k}^{T}(t)} H_{1} e_{l}(t)+\overline{a_{k l} e_{l}^{T}(t) H_{1}} e_{k}(t)\right. \\
& \left.+b_{k l} \overline{e_{k}^{T}(t)} H_{2} e_{l}(t)+b_{k l} \overline{e_{l}^{T}(t)} H_{2} e_{k}(t)\right) \\
\leq & M \sum_{k=1}^{N} \overline{e_{k}^{T}(t)} e_{k}(t)+c \overline{e^{T}(t)}\left(\left(A \otimes H_{1}+\overline{A^{T}} \otimes \overline{H_{1}}\right)+\left(B+B^{T}\right) \otimes H_{2}\right) e(t) \\
\leq & (M+\lambda) V(t)
\end{aligned}
$$

which gives

$$
V(t) \leq V\left(t_{\sigma-1}^{+}\right) e^{(M+\lambda)\left(t-t_{\sigma-1}\right)} .
$$

When $t=t_{\sigma}$, one has

$$
\begin{aligned}
V\left(t_{\sigma}^{+}\right) & =\sum_{k=1}^{N} \overline{e_{k}^{T}\left(t_{\sigma}^{+}\right)} e_{k}\left(t_{\sigma}^{+}\right)=\sum_{k \in P\left(t_{\sigma}\right)} \overline{e_{k}^{T}\left(t_{\sigma}^{+}\right)} e_{k}\left(t_{\sigma}^{+}\right)+\sum_{k \notin P\left(t_{\sigma}\right)} \overline{e_{k}^{T}\left(t_{\sigma}^{+}\right)} e_{k}\left(t_{\sigma}^{+}\right) \\
& =(1+b)^{2} \sum_{k \in P\left(t_{\sigma}\right)} \overline{e_{k}^{T}\left(t_{\sigma}^{-}\right)} e_{k}\left(t_{\sigma}^{-}\right)+\sum_{k \notin P\left(t_{\sigma}\right)} \overline{e_{k}^{T}\left(t_{\sigma}^{-}\right)} e_{k}\left(t_{\sigma}^{-}\right) \\
& =\beta \sum_{k=1}^{N} \overline{e_{k}^{T}\left(t_{\sigma}^{-}\right)} e_{k}\left(t_{\sigma}^{-}\right)+(1-\beta) \sum_{k \notin P\left(t_{\sigma}\right)} \overline{e_{k}^{T}\left(t_{\sigma}^{-}\right)} e_{k}\left(t_{\sigma}^{-}\right) .
\end{aligned}
$$

According to the definition of $P\left(t_{\sigma}\right)$, one has

$$
\frac{1}{N-p} \sum_{k \notin P\left(t_{\sigma}\right)} \overline{e_{k}^{T}\left(t_{\sigma}^{-}\right)} e_{k}\left(t_{\sigma}^{-}\right) \leq \frac{1}{N} \sum_{k=1}^{N} \overline{e_{k}^{T}\left(t_{\sigma}^{-}\right)} e_{k}\left(t_{\sigma}^{-}\right)
$$

and

$$
V\left(t_{\sigma}^{+}\right) \leq \beta \sum_{k=1}^{N} \overline{e_{k}^{T}\left(t_{\sigma}^{-}\right)} e_{k}\left(t_{\sigma}^{-}\right)+\frac{(N-p)(1-\beta)}{N} \sum_{k=1}^{N} \overline{e_{k}^{T}\left(t_{\sigma}^{-}\right)} e_{k}\left(t_{\sigma}^{-}\right)=\rho\left(t_{\sigma}\right) V\left(t_{\sigma}^{-}\right) .
$$

According to mathematical induction, combining inequalities (6) and (7), for any positive integer $\sigma$, the following inequality can be derived:

$$
V\left(t_{\sigma}^{+}\right) \leq V\left(t_{0}^{+}\right) \prod_{s=1}^{\sigma} \rho\left(t_{\sigma}\right) e^{(M+\lambda)\left(t_{s}-t_{s-1}\right)} .
$$

If conditions (5) hold, one has

$$
\rho\left(t_{\sigma}\right) e^{(M+\lambda)\left(t_{s}-t_{s-1}\right)}<e^{-\zeta}, s=1,2, \ldots, \sigma,
$$

and

$$
V\left(t_{\sigma}^{+}\right) \leq V\left(t_{0}^{+}\right) e^{-\sigma \zeta},
$$

which implies $V\left(t_{\sigma}^{+}\right) \rightarrow 0$ as $\sigma \rightarrow+\infty$. Then for $t \in\left(t_{\sigma}, t_{\sigma+1}\right)$, one has $V(t) \leq V\left(t_{\sigma}^{+}\right) e^{(M+\lambda)\left(t-t_{\sigma}\right)^{\alpha}} \rightarrow 0$ as $t \rightarrow+\infty$. which implies that $\left\|e_{k}(t)\right\| \rightarrow 0$ as $t \rightarrow+\infty$, 
i.e., the outer synchronization of drive-response networks (1) and (2) is achieved. This completes the proof.

Remark 1. From conditions (5), the necessary values of impulsive intervals and gains for achieving outer synchronization can be calculated for any given drive-response networks. For other drive-response networks with different system parameters, however, the necessary values must be recalculated. Thus, in the following, we combine adaptive strategy with impulsive control to design unified controllers.

Theorem 2. Suppose that Assumption 1 holds. If there exists a constant $\zeta>0$ such that

$$
\ln \rho\left(t_{\sigma}\right)+\zeta+\widehat{L}\left(t_{\sigma}\right) \tau_{\sigma}<0, \sigma=1,2, \cdots,
$$

holds, where $\widehat{L}(t)$ is the estimated value of $M+\lambda, \dot{\widehat{L}}(t)=\eta \overline{e^{T}(t)} e(t)$ and $\eta>0$ is adaptive gain, then the drive-response networks (1) and (2) achieve the outer synchronization.

Proof. Consider the following Lyapunov function:

$$
V(t)=\sum_{k=1}^{N} \frac{e_{k}^{T}(t)}{e_{k}}(t)+\frac{\rho(t)}{2 \eta}(\widehat{L}(t)-M-\lambda)^{2} .
$$

for $t \in\left(t_{\sigma-1}, t_{\sigma}\right], \sigma=1,2, \cdots$.

When $t \in\left(t_{\sigma-1}, t_{\sigma}\right)$, one has

$$
\begin{aligned}
\dot{V}(t)= & \sum_{k=1}^{N} \overline{e_{k}^{T}(t)} \dot{e}_{k}(t)+\sum_{k=1}^{N} \overline{\dot{e}_{k}^{T}(t)} e_{k}(t)+\frac{1}{\eta}(\widehat{L}(t)-M-\lambda) \dot{\widehat{L}}(t) \\
= & \left.\sum_{k=1}^{N} \overline{\left(e_{k}^{T}(t)\right.}\left(f\left(y_{k}(t)\right)-f\left(x_{k}(t)\right)\right)+\overline{\left(f\left(y_{k}(t)\right)-f\left(x_{k}(t)\right)\right)^{T}} e_{k}(t)\right) \\
& +c \sum_{k=1}^{N} \sum_{l=1}^{N}\left(a_{k l} \overline{e_{k}^{T}(t)} H_{1} e_{l}(t)+\overline{a_{k l} e_{l}^{T}(t) H_{1}} e_{k}(t)+b_{k l} \overline{e_{k}^{T}(t)} H_{2} e_{l}(t)\right. \\
& \left.+b_{k l} \overline{e_{l}^{T}(t)} H_{2} e_{k}(t)\right)+(\widehat{L}(t)-M-\lambda) \overline{e^{T}(t)} e(t) \\
\leq & \widehat{L}(t) \overline{e^{T}(t)} e(t) \\
\leq & \widehat{L}\left(t_{\sigma}\right) V(t),
\end{aligned}
$$

which gives

$$
V(t) \leq V\left(t_{\sigma-1}^{+}\right) e^{\widehat{L}\left(t_{\sigma}\right)\left(t-t_{\sigma-1}\right)}
$$

When $t=t_{\sigma}$,

$$
\begin{aligned}
V\left(t_{\sigma}^{+}\right) & =\sum_{k=1}^{N} \overline{e_{k}^{T}\left(t_{\sigma}^{+}\right)} e_{k}\left(t_{\sigma}^{+}\right)+\frac{\rho\left(t_{\sigma}\right)}{2 \eta}\left(\widehat{L}\left(t_{\sigma}\right)-M-\lambda\right)^{2} \\
& \leq \rho\left(t_{\sigma}\right) \sum_{k=1}^{N} \overline{e_{k}^{T}\left(t_{\sigma}^{-}\right)} e_{k}\left(t_{\sigma}^{-}\right)+\frac{\rho\left(t_{\sigma}\right)}{2 \eta}\left(\widehat{L}\left(t_{\sigma}\right)-M-\lambda\right)^{2} \\
& =\rho\left(t_{\sigma}\right) V\left(t_{\sigma}^{-}\right) .
\end{aligned}
$$

Thus, similar to the proof of Theorem 1, the proof can be completed.

Remark 2. From the conditions (8), when $p, N, b$ and $\zeta$ are fixed, the impulsive instants $t_{\sigma}$ can be chosen through finding the maximum value of $t_{\sigma}$ subject to $t_{\sigma}<t_{\sigma-1}-\left(\ln \rho\left(t_{\sigma}\right)+\zeta\right) \hat{L}^{-1}\left(t_{\sigma}\right)$ with $\sigma=1,2, \cdots$. 


\section{Numerical Simulations}

Example 1. Consider drive-response networks coupled with 10 nodes. Choose the node dynamics as a complex-variable Chen system [37]

$$
\begin{aligned}
& \dot{x}_{k 1}=27\left(x_{k 2}-x_{k 1}\right), \\
& \dot{x}_{k 2}=-4 x_{k 1}+23 x_{k 2}-x_{k 1} x_{k 3}, \\
& \dot{x}_{k 3}=\left(\overline{x_{k 1}} x_{k 2}+x_{k 1} \overline{x_{k 2}}\right) / 2-x_{k 3},
\end{aligned}
$$

where $x_{k 1}$ and $x_{k 2}$ are complex variables, $x_{k 3}$ is the real variable. Refer to [34], choose $M=79$ such that Assumption 1 holds.

Choose $c=1, H_{1}=\operatorname{diag}(1,1,0), H_{2}=\operatorname{diag}(0,0,1)$,

$$
A=\left[\begin{array}{cccccccccc}
-1-3 i & 0 & 0 & 0 & 0 & 0 & 0 & i & 0 & 1+2 i \\
1+i & -2-4 i & 0 & 0 & 0 & 0 & 0 & 0 & 1+3 i & 0 \\
0 & 0 & -3-2 i & 0 & 0 & 3+2 i & 0 & 0 & 0 & 0 \\
0 & 0 & 2+i & -3-5 i & 0 & 0 & 1+4 i & 0 & 0 & 0 \\
0 & 0 & 0 & 2+2 i & -2-2 i & 0 & 0 & 0 & 0 & 0 \\
0 & 0 & 0 & 0 & 1+i & -1-i & 0 & 0 & 0 & 0 \\
0 & 0 & 0 & 0 & 0 & 0 & -1-4 i & 0 & 0 & 1+4 i \\
3+4 i & -1-i & 0 & 0 & 0 & 0 & 0 & -2-3 i & 0 & 0 \\
0 & 5+4 i & 0 & -1+2 i & 0 & 0 & 0 & 0 & -4-6 i & 0 \\
0 & 0 & 0 & 2+i & 0 & 0 & 0 & 1+2 i & 0 & -3-3 i
\end{array}\right],
$$

$$
B=\left[\begin{array}{cccccccccc}
-4 & 0 & 0 & 0 & 0 & 0 & 0 & 1 & 0 & 3 \\
1 & -3 & 0 & 0 & 0 & 0 & 1 & 0 & 2 & 0 \\
0 & 0 & -5 & 0 & 0 & 5 & 0 & 0 & 0 & 0 \\
0 & 0 & 2 & -4 & 0 & 0 & 2 & 0 & 0 & 0 \\
0 & 0 & 0 & 3 & -3 & 0 & 0 & 0 & 0 & 0 \\
0 & 0 & 0 & 0 & 2 & -2 & 0 & 0 & 0 & 0 \\
0 & 0 & 0 & 0 & 0 & 0 & -3 & 0 & 0 & 3 \\
1 & 1 & 0 & 0 & 0 & 0 & 0 & -2 & 0 & 0 \\
0 & 5 & 0 & -1 & 0 & 0 & 0 & 0 & -4 & 0 \\
0 & 0 & 0 & 2 & 0 & 0 & 0 & 3 & 0 & -5
\end{array}\right]
$$

In numerical simulations, choose $b=-0.9, \zeta=0.01, p=3$ and $\tau_{\sigma}=0.004$ for $\sigma=$ $1,2, \cdots$. By simple calculations, one has $\lambda=2.2253$ and $\ln 0.703+0.01+(M+\lambda) \times(0.004)=$ $-0.017499<0$. That is, the conditions (5) in Theorem 1 are satisfied and the outer synchronization can be achieved. Choose the initial values of $x_{k}(0)=0.1 \times(2+0.2 k i, 1+0.5 k i, 0.1 k)^{T}$ and $y_{k}(0)=(1+0.5 k i, 2+0.5 k i, 0.2 k)^{T}$ with $i=\sqrt{-1}$ for $k=1,2, \cdots, 10$. Figure 1 shows the orbits of the norms of synchronization errors $e_{k}(t), k=1,2, \cdots, 10$.

Example 2. Consider the same network in Example 1 via adaptive impulsive pinning control. In numerical simulations, choose $b=-0.9, \eta=0.0002, \hat{L}(0)=0.1$. The impulsive instants are estimated according to Remark 2. Figure 2 shows the orbits of the norm of synchronization errors. Figure 3 shows the impulsive interval $\tau_{\sigma}$ versus $\sigma$. From Figure 3 , it can be seen that the necessary value of $\tau_{\sigma}$ is much larger than the estimated value from conditions (5), i.e., the adaptive impulsive pinning control can reduce control cost.

Example 3. Consider the outer synchronization of drive-response network consisting of 10 Lü systems [37]

$$
\begin{aligned}
& \dot{x}_{k 1}=40\left(x_{k 2}-x_{k 1}\right), \\
& \dot{x}_{k 2}=-x_{k 1} x_{k 3}+22 x_{k 2}, \\
& \dot{x}_{k 3}=\left(\overline{x_{k 1}} x_{k 2}+x_{k 1} \overline{x_{k 2}}\right) / 2-5 x_{k 3},
\end{aligned}
$$

where $x_{k 1}$ and $x_{k 2}$ are complex variables, $x_{k 3}$ is the real variable. In numerical simulations, choose $b=-0.9, \eta=0.003, \hat{L}(0)=0.1$ with the other parameters the same as in Example 1. Figure 4 
shows the orbits of the norm of synchronization errors. Figure 5 shows the impulsive interval $\tau_{\sigma}$ versus $\sigma$.

From Examples 2 and 3, the adaptive impulsive pinning controllers are valid for different networks. That is, the controllers are universal to some extent.

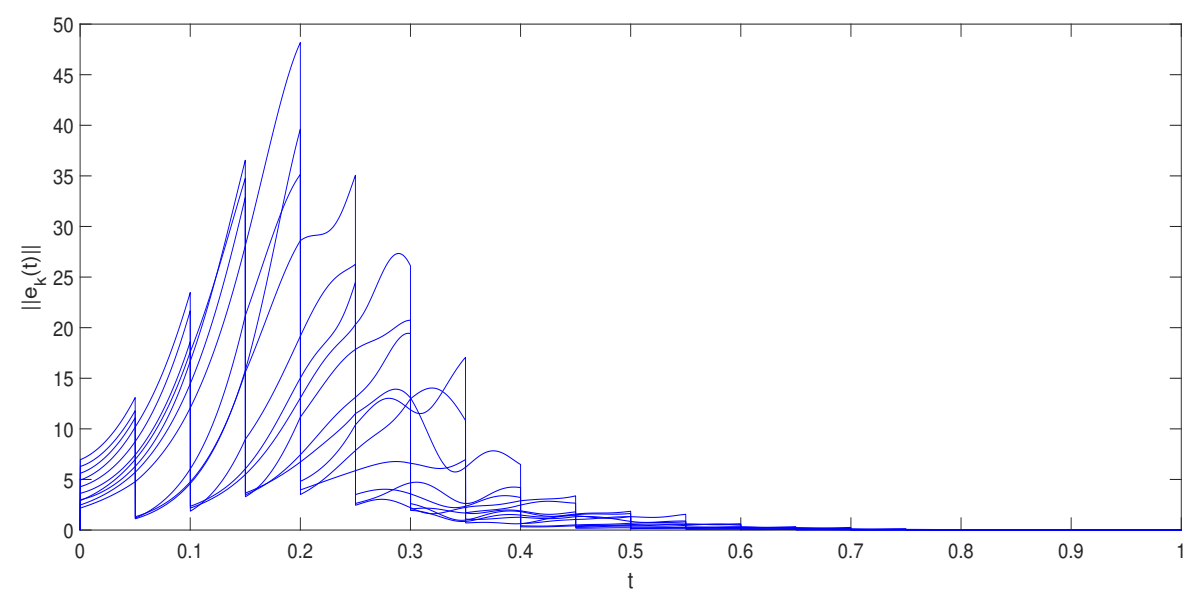

Figure 1. The orbits of the norm of synchronization errors $\left\|e_{k}(t)\right\|$.

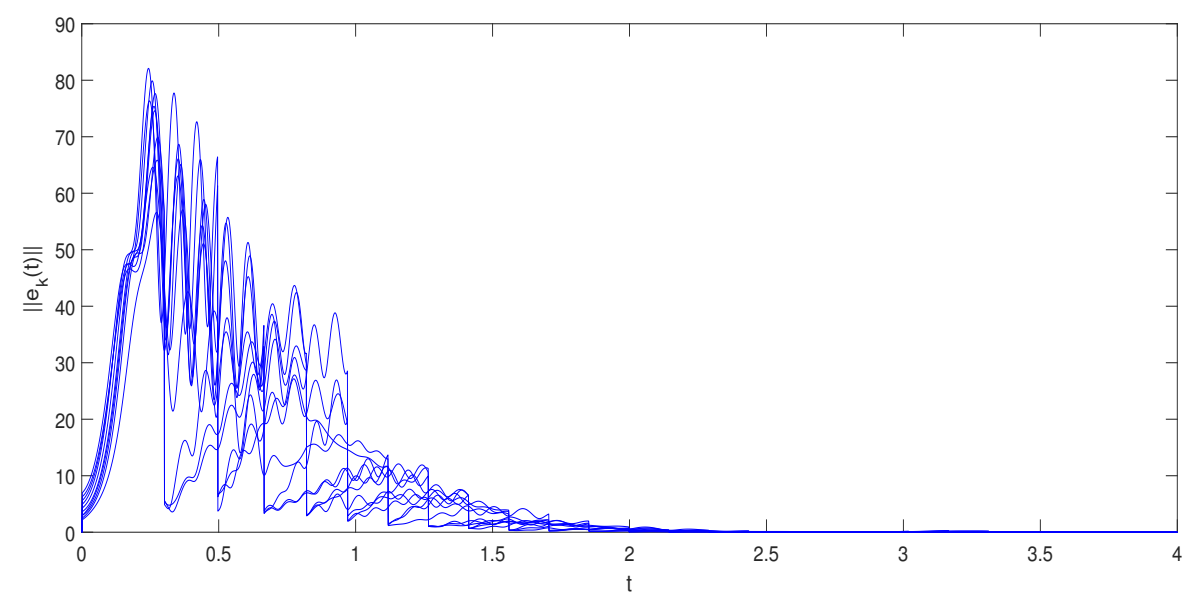

Figure 2. The orbits of the norm of synchronization errors $\left\|e_{k}(t)\right\|$.

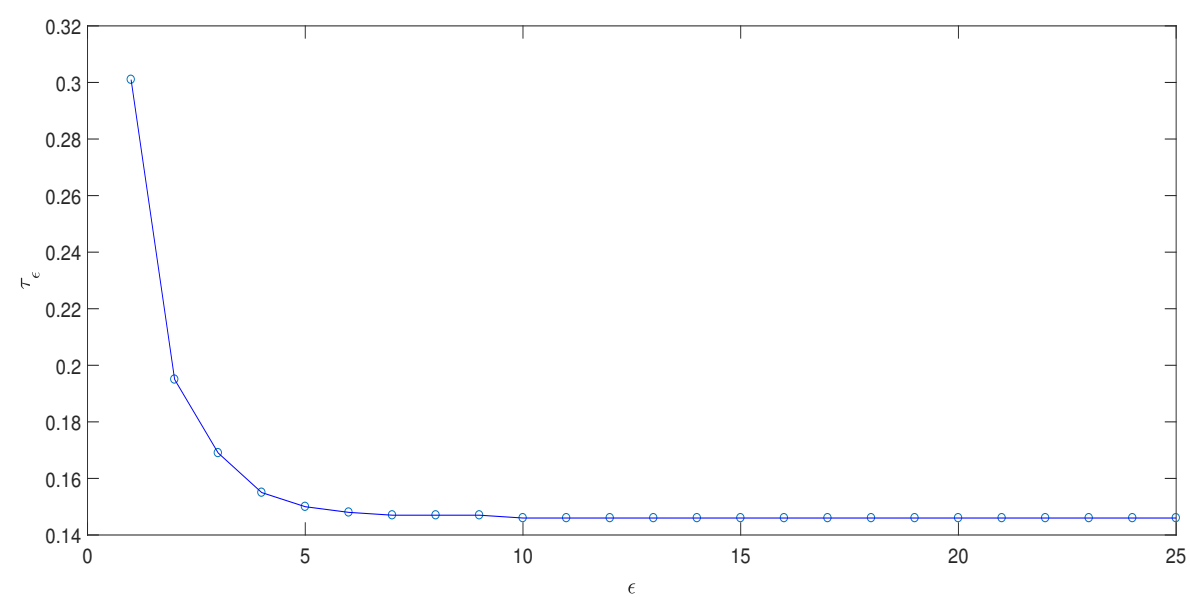

Figure 3. The orbit of $\tau_{\sigma}$ vs. $\sigma$. 


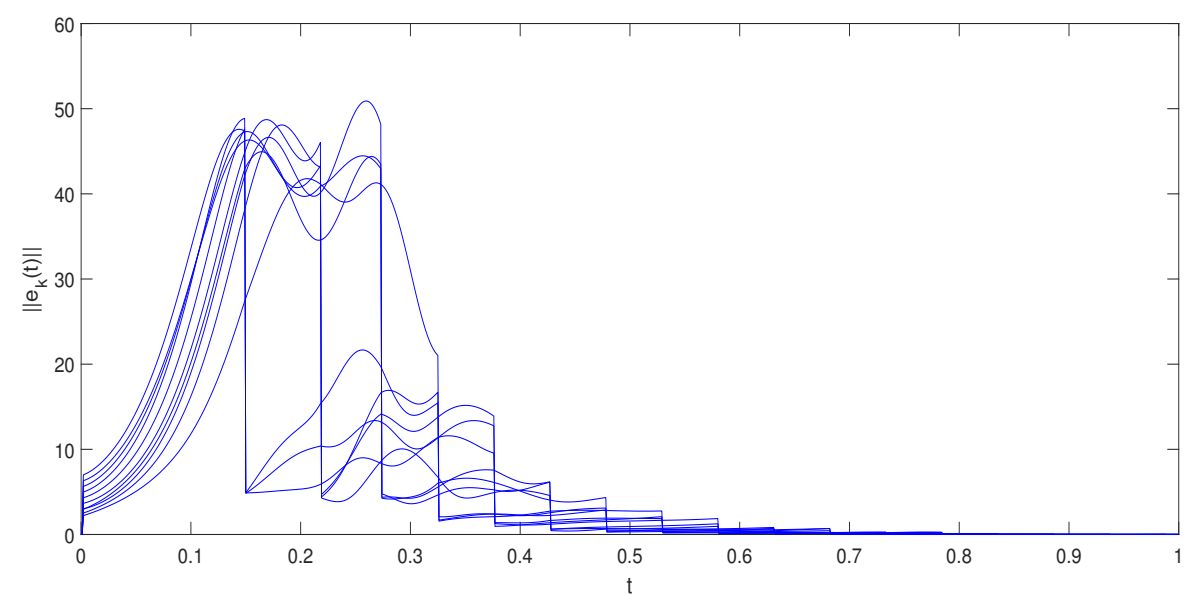

Figure 4. The orbits of the norm of synchronization errors $\left\|e_{k}(t)\right\|$.

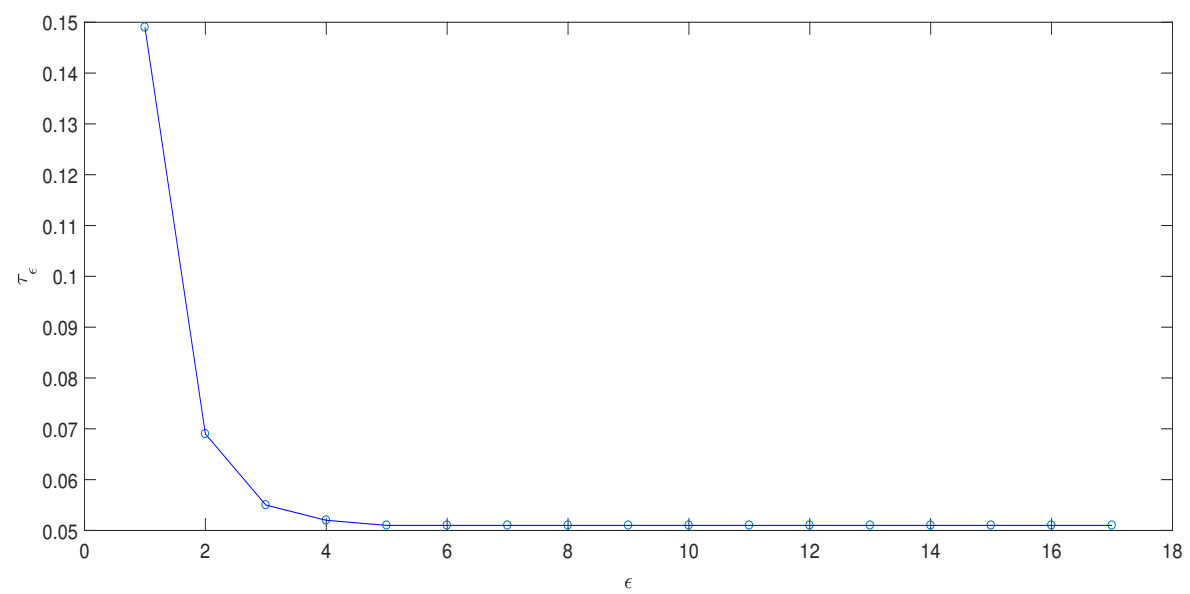

Figure 5. The orbit of $\tau_{\sigma}$ vs. $\sigma$.

\section{Conclusions and Discussions}

Both static and adaptive impulsive pinning controllers are designed for achieving the outer synchronization of complex-variable networks with complex coupling. The corresponding synchronization conditions and estimation algorithms with respect to the impulsive gains and intervals are provided as well. Based on the Lyapunov function method and stability theory of impulsive differential equations, the main results are analytically proved. The obtained results are illustrated to be effective by three numerical examples.

On the other hand, synchronization and control of fractional-order dynamical networks attracts increasing attention [5-8]. As we know, the fractional-order system has a long memory effect from the initial state to the current state. When the impulsive controllers are added at $t=t_{k}$, the memory effect for $t \in\left(t_{k}, t_{k+1}\right)$ needs to be adjusted from $t_{k}$ to $t$. That is, the obtained results in this paper cannot be directly extended to the fractional-order dynamical networks. Thus, designing impulsive (or hybrid impulsive) controllers for fractional-order dynamical networks is a challenging and important issue and deserves further studies.

Author Contributions: Y.J. writes the original draft preparation, Z.W. reviews and edits the whole paper. Both authors have read and agreed to the published version of the manuscript.

Funding: This work was jointly supported by the NSFC Grant Nos. 61963019 and 61463022 , the NSF for Distinguished Young Scholar of Jiangxi Province of China Grant No. 20171BCB23031 and the Graduate Innovation Fund of Jiangxi Province of China Grant No. YC2020-S187.

Institutional Review Board Statement: Not applicable. 
Informed Consent Statement: Not applicable.

Data Availability Statement: The data can be found in the manuscript.

Conflicts of Interest: The authors declare no conflict of interest.

\section{References}

1. Du, W.J.; Zhang, J.G.; An, X.L.; Qin, S.; Yu, J.N. Outer Synchronization between Two Coupled Complex Networks and Its Application in Public Traffic Supernetwork. Discret. Dyn. Nat. Soc. 2016, 2016, 8920764. [CrossRef]

2. Zhou, L.; Wang, C.; He, H.; Lin, Y. Time-controllable combinatorial inner synchronization and outer synchronization of anti-star networks and its application in secure communication. Commun. Nonlinear Sci. Numer. Simul. 2015, 22, 623-640. [CrossRef]

3. Geng, L.; Xiao, R. Outer synchronization and parameter identification approach to the resilient recovery of supply network with uncertainty. Phys. A Stat. Mech. Appl. 2017, 482, 407-421. [CrossRef]

4. Zhang, L.; Lei, Y.; Wang, Y.; Chen, H. Generalized outer synchronization between non-dissipatively coupled complex networks with different-dimensional nodes. Appl. Math. Model. 2018, 55, 248-261. [CrossRef]

5. Ma, T.; Zhang, J. Hybrid synchronization of coupled fractional-order complex networks. Neurocomputing 2015, 157, 166-172. [CrossRef]

6. Asheghan, M.M.; Míguez, J.; Hamidi-Beheshti, M.T.; Tavazoei, M.S. Robust outer synchronization between two complex networks with fractional order dynamics. Chaos 2011, 21, 033121. [CrossRef]

7. Zhao, M.; Wang, J. Outer synchronization between fractional-order complex networks: A non-fragile observer-based control scheme. Entropy 2013, 15, 1357-1374. [CrossRef]

8. Yang, Y.; Wang, Y.; Li, T. Outer synchronization of fractional-order complex dynamical networks. Optik 2016, 127, 7395-7407. [CrossRef]

9. Li, C.; Lü, L.; Yang, Y.; Zhou, S.; Hong, Y. Research on outer synchronization between uncertain time-varying networks with different node number. Phys. A Stat. Mech. Appl. 2018, 492, 2301-2309. [CrossRef]

10. Arellano-Delgado, A.; López-Gutiérrez, R.M.; Méndez-Ramírez, R.; Cardoza-Avendaño, L.; Cruz-Hernández, C. Dynamic coupling in small-world outer synchronization of chaotic networks. Phys. D Nonlinear Phenom. 2021, 423, 132928. [CrossRef]

11. Li, C.; Sun, W.; Kurths, J. Synchronization between two coupled complex networks. Phys. Rev. E Stat. Nonlinear Soft Matter Phys. 2007, 76, 046204. [CrossRef] [PubMed]

12. Li, C.; Xu, C.; Sun, W.; Xu, J.; Kurths, J. Outer synchronization of coupled discrete-time networks. Chaos 2009, 19, 013106. [CrossRef]

13. $\mathrm{Wu}, \mathrm{Z} . Y$. Adaptive impulsive outer synchronization between drive-response dynamical networks. Commun. Theor. Phys. 2014, 61, 590-594. [CrossRef]

14. Wu, Z.; Chen, G.; Fu, X. Outer synchronization of drive-response dynamical networks via adaptive impulsive pinning control. $J$. Frankl. Inst. 2015, 352, 4297-4308. [CrossRef]

15. Cai, S.; Lei, X.; Liu, Z. Outer synchronization between two hybrid-coupled delayed dynamical networks via aperiodically adaptive intermittent pinning control. Complexity 2016, 21, 593-605. [CrossRef]

16. Lei, X.; Cai, S.; Jiang, S.; Liu, Z. Adaptive outer synchronization between two complex delayed dynamical networks via aperiodically intermittent pinning control. Neurocomputing 2017, 222, 26-35. [CrossRef]

17. Li, X.; Wang, N.; Lu, J.; Alsaadi, F.E. Pinning outer synchronization of partially coupled dynamical networks with complex inner coupling matrices. Phys. A Stat. Mech. Appl. 2019, 515, 497-509. [CrossRef]

18. Sheng, S.; Zhang, X.; Lu, G. Finite-time outer-synchronization for complex networks with Markov jump topology via hybrid control and its application to image encryption. J. Frankl. Inst. 2018, 355, 6493-6519. [CrossRef]

19. Zhang, Z.; Wang, H.; Xu, F.; Jin, Y.Q. Complex-valued convolutional neural network and its application in polarimetric SAR image classification. IEEE Trans. Geosci. Remote Sens. 2017, 55, 7177-7188. [CrossRef]

20. Quan, Y.; Chen, Y.; Shao, Y.; Teng, H.; Xu, Y.; Ji, H. Image denoising using complex-valued deep CNN. Pattern Recognit. 2021, 111, 107639. [CrossRef]

21. Hirose, A. Complex-Valued Neural Networks: Advances and Applications; John Wiley \& Sons: Hoboken, NJ, USA, 2013. [CrossRef]

22. Gibbon, J.D.; McGuinness, M.J. The real and complex Lorenz equations in rotating fluids and lasers. Phys. D Nonlinear Phenom. 1982, 5, 108-122. [CrossRef]

23. Ning, C.Z.; Haken, H. Detuned lasers and the complex Lorenz equations: Subcritical and supercritical Hopf bifurcations. Phys. Rev. A 1990, 41, 3826-3837. [CrossRef]

24. Fowler, A.C.; Gibbon, J.D.; McGuinness, M.J. The real and complex Lorenz equations and their relevance to physical systems. Phys. D Nonlinear Phenom. 1983, 7, 126-134. [CrossRef]

25. Pan, J.; Zhang, Z. Finite-time synchronization for delayed complex-valued neural networks via the exponential-type controllers of time variable. Chaos Solitons Fractals 2021, 146, 110897. [CrossRef]

26. Xu, Y.; Zhou, W.; Fang, J.; Sun, W.; Pan, L. Adaptive synchronization of stochastic time-varying delay dynamical networks with complex-variable systems. Nonlinear Dyn. 2015, 81, 1717-1726. [CrossRef]

27. Hou, T.; Yu, J.; Hu, C.; Jiang, H. Finite-time synchronization of fractional-order complex-variable dynamic networks. IEEE Trans. Syst. Man Cybern. Syst. 2019, 51, 4297-4307. [CrossRef] 
28. Wu, Z.; Leng, H. Complex hybrid projective synchronization of complex-variable dynamical networks via open-plus-closed-loop control. J. Frankl. Inst. 2017, 354, 689-701. [CrossRef]

29. Liu, D.; Zhu, S.; Sun, K. New results for exponential stability of complex-valued memristive neural networks with variable delays. Neurocomputing 2018, 275, 758-767. [CrossRef]

30. Wu, Z.; Liu, D.; Ye, Q. Pinning impulsive synchronization of complex-variable dynamical network. Commun. Nonlinear Sci. Numer. Simul. 2015, 20, 273-280. [CrossRef]

31. Leng, $\mathrm{H}$.; Wu, Z. Impulsive synchronization of complex-variable network with distributed time delays. Phys. A Stat. Mech. Appl. 2019, 536, 122602. [CrossRef]

32. Yang, S.; Hu, C.; Yu, J.; Jiang, H. Finite-Time Cluster Synchronization in Complex-Variable Networks with Fractional-Order and Nonlinear Coupling. Neural Netw. 2021, 135, 212-224. [CrossRef] [PubMed]

33. Zheng, S. Impulsive complex projective synchronization in drive-response complex coupled dynamical networks. Nonlinear Dyn. 2015, 79, 147-161. [CrossRef]

34. Wu, Z.; Chen, G.; Fu, X. Synchronization of a network coupled with complex-variable chaotic systems. Chaos 2012, 22, 023127. [CrossRef]

35. Wu, X.; Feng, J.; Nie, Z. Outer synchronization of drive-response complex-valued complex networks via intermittent pinning control. Complexity 2021, 2021, 6649519. [CrossRef]

36. $\mathrm{Wu}, \mathrm{Z} . ; \mathrm{Fu}, \mathrm{X}$. Synchronization of complex-variable dynamical networks with complex coupling. Int. J. Mod. Phys. C 2013, 24, 1350007. [CrossRef]

37. Mahmoud, G.M.; Bountis, T.; Mahmoud, E.E. Active control and global synchronization of the complex chen and Lü systems. Int. J. Bifurc. Chaos 2007, 17, 4295-4308. [CrossRef] 